\title{
SEARCH DARK MATTER IN THE GALAXY
}

\author{
A.A. Mikhailov \\ Yu.G. Shafer Institute of Cosmophysical Research and Aeronomy \\ 31 Lenin Ave., 677980 Yakutsk, Russia \\ E-mail: mikh@ikfia.ysn.ru
}

We analyzed of Yakutsk EAS array data and found 14 particles which come from center of Galaxy to Earth without any deviation in galactic magnetic field. We suppose that these particles are particles of dark matter. 


\section{Introduction}

We analyzed distribution particles in celestial sphere by data Yakutsk array at energy $\mathrm{E} \geq 8 \times 10^{18} \mathrm{eV}$. It is consider arrival directions of extensive air shower (EAS) or particles with zenith angles $\theta<60^{\circ}$ and cores located inside the array perimeter. We consider primary particles with energies $\mathrm{E} \geq 5 \times 10^{18} \mathrm{eV}$. Energy of particles determined by registration Cherenkov light with accuracy's $\sim 30 \%$ and angles $\sim 5-7^{\circ}$.

\subsection{The Distribution Particles in Right Ascension}

We consider Yakutsk array data at energy $\mathrm{E} \geq 5 \times 10^{18} \mathrm{eV}$ including from 1974 until 2011. At first we analyzed by harmonic analyses distribution particles in right ascension: at energy $\mathrm{E} \geq 8 \times 10^{18} \mathrm{eV}$ amplitude and phase in right ascension of 1-st harmonic of Fourier analyses is $r_{1}=0.14 \pm 0.04, \varphi_{1}=0.6^{\circ} \pm 19^{\circ}, r_{2}=0.02 \pm 0.04, \varphi_{2}=8^{\circ} \pm 125^{\circ}$, number of particles $n=938$; chance probability $\mathrm{P}$ have amplitudes $\mathrm{r}_{1} \geq 0.14$ is $\mathrm{P} \sim 0.01$.

Early we suggest new method of analyzes data only along in two coordinate, declination and right ascension and we analyses by this method data [1]. We did not found statistically significance amplitude of particles directions by Fourier analyses, but phases of 1-st harmonic at energy $E>10^{19} \mathrm{eV}$ start change to right ascension RA $180^{\circ}$ or to high galactic latitudes b (Fig.1). This change phase of 1-st harmonics most likely point to change sources of ultrahigh energy cosmic rays to high latitudes.

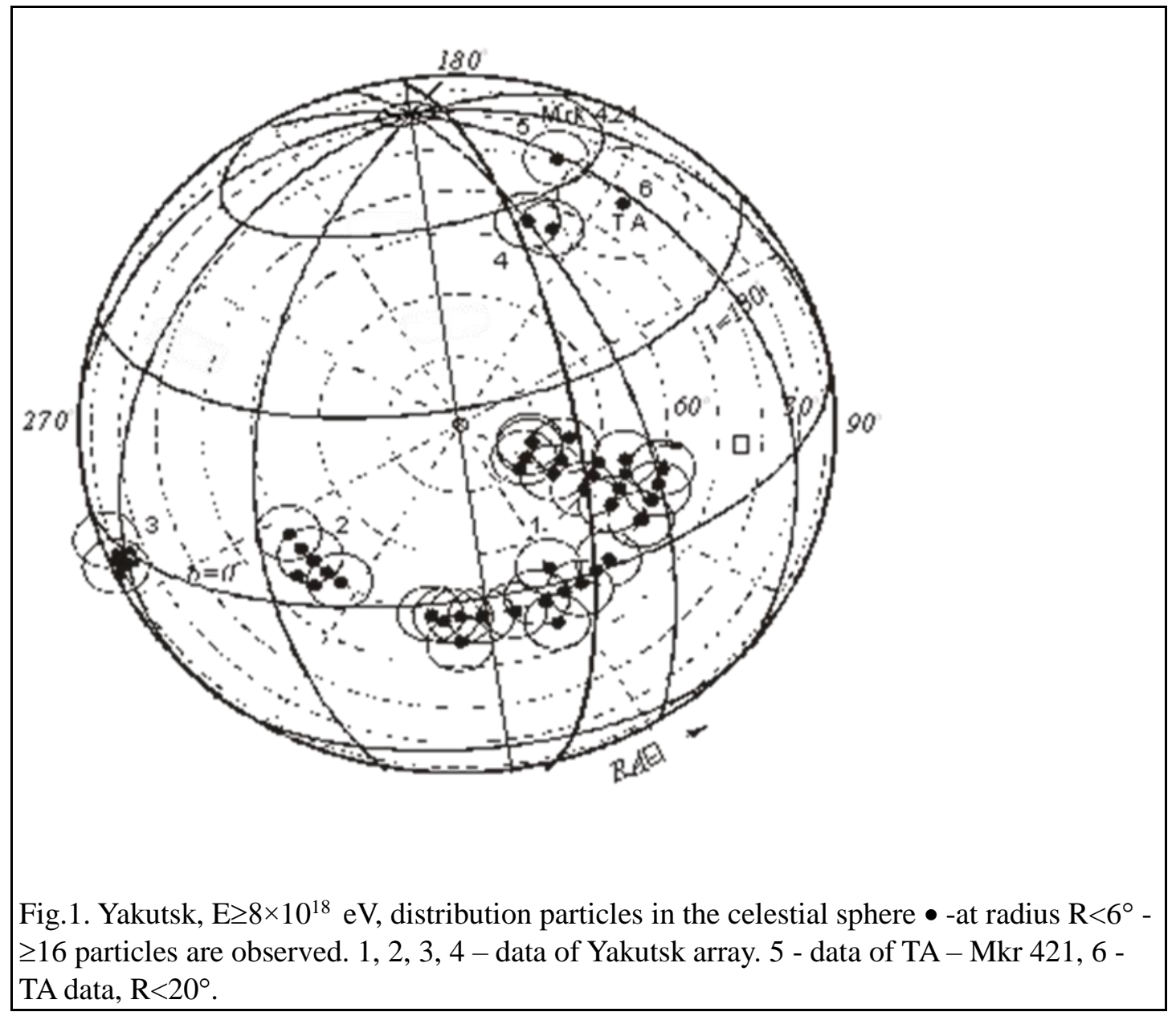




\subsection{The distribution particles energies $\mathrm{E}>8 \times 10^{18} \mathrm{eV}$ in celestial sphere}

In previous paper [1] we conclude that cosmic rays until energy $\mathrm{E}<4 \times 10^{19} \mathrm{eV}$ are galactic and their sources are pulsars. Here we have found 6 regions (include data TA), inside the 1-st region (Fig.1) - 39 pulsars, 2-nd region - 16 pulsars, 3-rd region >10 pulsars (Galactic center), 4th region -4 pulsars inside these regions $183,11,17,23,14,11$ particles accordingly. The chance probabilities to find such number of events from 938 particles are equal: $P_{1}(938, \geq 183) \sim 6.10^{-5}$, $\mathrm{P}_{2}(938, \geq 11) \sim 0.6, \mathrm{P}_{3}(938, \geq 17), \sim 2.10^{-3}, \mathrm{P}_{4}(938, \geq 13) \sim 0.5, \mathrm{P}_{4,5} \sim 0.1$.

Note that chance probabilities significant only around 1-st and 3-d groups of pulsars at radius $\mathrm{R}<6^{\circ}$ are $>3 \sigma$ than expected in a case isotropy. The maximum number of events $20 \leq n \leq 23$ at $R<6^{\circ}$ are observed around the 1-st groups of pulsars: PSR 0141+6009, 0146+6145, 0147+5922, $0157+6212,0215+6218$. The given pulsars are in galactic plane and have been considered earlier as possible sources of particles early [1].

It is observed flux particles from side center of Galaxy (Fig.1, number 3). All 14 particles come under large zenith angles $60^{\circ} \geq \theta \geq 38^{\circ}$ to Yakutsk array and come from only one direction as neutral particles of dark matter (trajectory of these particles not similar other particles - come directly from one direction - Galactic center without any deviation). From 6 showers we have no any information about muon component data - these showers registered in summer (sometimes in summer some muon detectors did not operate because muon detectors are underground in permafrost and possible melt permafrost).

At autumn after repair muon detectors begin to operate. Note from direction of Galactic center excess particles were found in main autumn at energy $\mathrm{E} \sim 10^{18} \mathrm{eV}$ (at this time it is wait according theory maximum fluxes particles from dark matter [2]).

We suppose that we registered particles of dark matter. Also AGASA and Sydney arrays informed that the registered particles come from side of Galactic center [3,4].

\section{CONCLUSION}

We have found correlation arrival directions of ultrahigh energy $\mathrm{E}<4 \times 10^{19} \mathrm{eV}$ particles with the positions of pulsars and anisotropy of arrival directions of these particles from side of the galactic plane by Yakutsk array data and Telescope array data at energy E $>5.7 \times 10^{19} \mathrm{eV}$ at high galactic latitudes. Our analyses of data show that observed cosmic rays up to $4 \times 10^{19} \mathrm{eV}$ are galactic and above this energy most likely extragalactic.

\section{References}

[1] A.A. Mikhailov, Analysis of the Arrival Directions of Ultrahigh Energy Particles, in book of Discovery of Cosmic Rays, ed. J.Ormes. Denver, June 26-28, 2012.

[2] V.A. Kuzmin, V.A. Rubakov, Yadernaya Fizika, 1998, 61, 1122.

[3] R. Anda, C. Aguirre, K. Tachi, in Proc.of 17-th ICRC, Paris,1981, 2, 164.

[4] A.D. Bray, L. Horton., C.B.A. McCusker, in Proc.17-th ICRC, 1981, 2, 168. 